\title{
On Solutions of Algebraic Differential Equations whose Coefficients are Analytic Functions in the Unit Disk $\left(^{*}\right)\left({ }^{* *}\right)$.
}

\author{
Steven B. BaNk (Urbana, Illinois)
}

Summary. - We investigate the growth of solutions for a class of first-order algebraic differential equations.

\section{1. - Introduction.}

In this paper, we investigate the rate of growth of analytic and meromorphic solutions of first order algebraic differential equations whose coefficients are analytic functions in the unit disk which are of finite order of growth (i.e., equations of the form $\Omega\left(z, y, y^{\prime}\right)=0$, where $\Omega\left(z, y, y^{\prime}\right)=\sum f_{k j}(z) y^{k}\left(y^{\prime}\right)^{j}$ is a polynomial in $y$ and $y^{\prime}$, and where for each $(k, j), f_{k j}(z)$ is an analytic function in the unit disk, whose order of growth,

$$
\limsup _{r \rightarrow 1}\left(\log \left[\log M\left(r, f_{k j}\right)\right] / \log \left[(1-r)^{-1}\right]\right)
$$

is finite).

In [8], VALIRoN treated the special case where all the coefficients $f_{k j}(z)$ are polynomials, and in this case, it was shown that any analytic solution in the unit disk must be of finite order of growth. In [2], it was shown that the same conclusion holds for the broader class of equations consisting of those whose coefficients are subject to the following restriction: If $p=\max \left\{k+j: f_{k j} \neq 0\right\}$, then $f_{k j}(z)$ is an analytic function of finite order in the unit disk if $k+j<p$, while if $k+j=p, f_{k j}(z)$ is a polynomial. The main technique used in the proof of the above results was ValironWiman theory for the unit disk [8; p. 299], which provides a relation between the maximum modulus and the logarithmic derivative of an analytic function of positive order in the disk, at certain points on a sequence of "remarkable " circles $|z|=r_{n}$ where $\lim _{n \rightarrow \infty} r_{n}=1$. In the case of the above mentioned results in $[2,8]$, this relation, for a function of infinite order in the disk, when used together with the differential equation, was sufficient to obtain a contradiction, thus showing that such functions cannot be solutions of such equations.

(*) Entrata in Redazione il 22 maggio 1971.

(**) This research was supported in part by the National Science Foundation (GP 19590) 
In the present paper, we treat the general case where the coefficients $f_{k j}(z)$ are arbitrary analytic functions of finite order in the unit disk. Of course, such equations can possess analytic solutions of infinite order in the disk (e.g., $\exp \left(\exp \left[(1-z)^{-1}\right]\right)$, $\left.\sin \left(\cos \left[(1-z)^{-1}\right]\right)\right)$, and hence the only information that the Valiron-Wiman theory can provide is an estimate on the maximum modulus of a solution on the sequence of "remarkable" circles, which of course is a very weak growth result. Instead, in this paper, we use the NEVANLINNA theory of meromorphic functions to obtain the following strong growth result (\$2) for analytic solutions in the disk: If $d$ is a positive real number which is greater than the order of each coefficient $f_{k j}(z)$ in the equation, then for any analytic solution $y_{0}(z)$ in the disk, there exists a constant $r_{0}$ in $[0,1)$ such that for all $r$ satisfying $r_{0}<r<1$, the maximum modulus of $y_{0}$ satisfies the inequality, $M\left(r, y_{0}\right) \leqslant \exp \left(\exp \left[(1-r)^{-(\dot{d}+4)}\right]\right)$.

In $\$ 6$, we use our main result to obtain growth estimates for certain meromorphio solutions in the unit disk. However, we point out here that for arbitrary meromorphic solutions in the disk, no such uniform growth estimate is possible, since it can be shown that for any increasing function $\Phi(r)$ on $[0,1)$, there exists a meromorphic solution of an equation in our class, whose NEVANLINNA characteristic dominates $\Phi(r)$ at a sequence of $r$ tending to 1 . (For more details, the reader is referred to the Remark in $\S 6$ ).

Finally, we briefly mention here that in an earlier paper by the author [1], an analogous result to our main theorem here was proved for entire solutions of equations where the coefficients $f_{h l}(z)$ are arbitrary entire functions. The main techniques in [1] were the BLUMENTaI theory of entire functions of infinite order and the VaLmonWIMAN theory for the plane [9]. The latter theory, in contrast to the corresponding theory for the disk, provides information at certain points on a "large " set of circles $|z|=r(i, e$., for all $r \geqslant 1$ with the possible exception of a set having finite logarithmic measure).

2. - We now state onr main result. The proof will be given in $\$ 5$.

THeonem. - Let $\Omega\left(z, y, y^{\prime}\right)=\sum f_{k j}(z) y^{k}\left(y^{\prime}\right)^{j}$ be a polynomial in $y$ and $y^{\prime}$, whose coefficients $f_{k j}(z)$ are analytic functions in the unit disk which are of finite order of growth. Let $d$ be a positive real number which is greater than the order of each coefficient $f_{k, j}(z)$. Then for any analytic function $y_{0}(z)$ in the unit disk, which satisfies $\Omega\left(z, y_{0}(z), y_{0}^{\prime}(z)\right) \equiv 0$, there exists a constant $r_{0}$ in $[0,1)$ such that

$$
M\left(r, y_{0}\right) \leqslant \exp \left(\exp \left[(1-r)^{-(d+4)}\right]\right),
$$

for all $r$ satisfying $r_{0}<r<1$.

\section{3. - Notation.}

For a meromorphic function $g(z)$ in the unit disk, we will use the standard notation for the NEVANLINNA functions $m(r, g), N(r, g)$ and $T(r, g)$ introduced in $[4 ; \mathrm{pp} .6,12]$. 


\section{4. - Lemma $A$.}

Let $f(z)$ be an analytic function in the unit disk which is not identically zero and which is of finite order $\leqslant \alpha$ in the disk. Then there exist a positive constant $K$ and a subset $F$ of $[0,1)$ such that,

$$
\int_{F}(1-r)^{-1} d r<+\infty
$$

and such that if $r$ belongs to $[0,1)$ and $r \notin F$, then

$$
|f(z)| \geqslant \exp \left(-K /(1-r)^{\alpha+4}\right) \text { on }|z|=r
$$

Proof. - Let $a_{1}, a_{2}, \ldots$ be the sequence of zeros of $f$ (if any) in $0<|z|<1$, arranged so that $\left|a_{1}\right| \leqslant\left|a_{2}\right| \leqslant \ldots$, and let $f$ have a $k$-fold zero at $z=0(k \geqslant 0)$. Let $\mu$ be the convergence exponent $[7 ; \mathrm{p} .7]$ of $\left\{a_{n}\right\}$, so

$$
\mu \leqslant \alpha .
$$

We now define an index $n_{0}$ and a set $F_{1}$ as follows:

If the sequence $\left\{a_{n}\right\}$ is empty, set $n_{0}=0$ and take $F_{1}$ to be the empty set. If the sequence $\left\{a_{n}\right\}$ is non-empty but finite, say $a_{1}, a_{2}, \ldots, a_{n}$, take $n_{0}=m$ and again let $F_{1}$ be empty. If the sequence $\left\{a_{n}\right\}$ is infinite, then $\lim _{n \rightarrow \infty}\left|a_{n}\right|=1$, so we may take $n_{0}$ to be an index such that,

$$
\left(1-\left|a_{n}\right|\right)^{\mu+8}\left(1+\left|a_{n}\right|\right)^{\mu+4} \leqslant \frac{1}{2} \quad \text { for all } n \geqslant n_{0} .
$$

In this case, let $F_{1}$ be the union (for $n>n_{0}$ ) of the closed intervals $I_{n}=\left[b_{n}, c_{n}\right]$, where $b_{n}=\left|a_{n}\right|-\left(1-\left|a_{n}\right|^{2}\right)^{n+4}$ and $c_{n}=\left|a_{n}\right|+\left(1-\left|a_{n}\right|^{2}\right)^{n+4}$.

We assert that in all cases,

$$
\int_{F_{1}}(1-r)^{-1} d r<+\infty
$$

This is clear if $F_{1}$ is empty. In the other case, we have,

$$
\int_{I_{n}}(1-r)^{-1} d r=\log \left(\left(1-b_{n}\right) /\left(1-c_{n}\right)\right) .
$$

Writing $\left(\left(1-b_{n}\right) /\left(1-c_{n}\right)\right)=1+w_{n}$, where $w_{n}=\left(c_{n}-b_{n}\right) /\left(1-c_{n}\right)$, and using the elementary inequality $\log (1+x) \leqslant x$, we obtain

$$
\int_{i_{n}}(1-r)^{-1} d r \leqslant w_{n} .
$$


Caleulating $w_{n}$ and cancelling the common factor $\left(1-\left|a_{n}\right|\right)$ from the numerator and denominator, and then using inequality (6), we clearly obtain,

$$
w_{n} \leqslant 4\left(1-\left|a_{n}\right|\right)^{\mu+3}\left(1+\left|a_{n}\right|\right)^{\mu+4} .
$$

Since $\left|a_{n}\right|<1$ and since $\sum_{n=1}^{\infty}\left(1-\left|a_{n}\right|\right)^{\mu+3}$ converges (by definition of convergence exponent), (7) now follows immediatly from (9) and (10).

Now let $Q(z)=z^{k} \prod_{n=1}^{n_{8}}\left(z-a_{n}\right)$. Then $f / Q$ is analytic in the disk and its sequence of zeros is $\left\{a_{n_{\mathrm{a}}+1}, \ldots\right\}$, which clearly has convergence exponent $\mu$. Let $\Phi(z)$ be the canonical product $[7 ;$ p. 7] formed with this sequence of zeros. Hence there is an analytic function $v(z)$ in the disk such that,

$$
f=Q \Phi e^{v}
$$

Now by $[7 ;$ p. 8$]$ (and $(5)), T(r, \Phi)=\bigcirc\left((1-r)^{-(\alpha+2)}\right)$, and by hypothesis, $T(r, f)=O\left((1-r)^{-(\alpha+1)}\right)$. Since $Q$ is of bounded characteristic (being a polynomial), it follows (11) and the usual rules $[4 ; \mathrm{p} .15]$ for calculating with the characteristic, that $T\left(r, e^{v}\right)=O\left((1-r)^{-(x+2)}\right)$. It thus follows [4; p. 140], that $\log ^{+} M\left(r, e^{v}\right)=$ $=O\left((1-r)^{-(\alpha+3)}\right)$. Thus $A(r)=\max _{\mid z=r}(\operatorname{Re} v(z))$ is $\bigcirc\left((1-r)^{-(\alpha+8)}\right)$. Hence by a theorem of CaRathÉodoRY $\left[6 ;\right.$ p. 338], $M(r, v)=O\left((1-r)^{-(\alpha+4)}\right)$. Thus there exist $K_{1}>0$ and $r_{1}$ in $[0,1)$ such that on $|z|=r$,

$$
\left|e^{v}\right| \geqslant \exp (-|v(z)|) \geqslant \exp \left(-K_{1} /(1-r)^{\alpha+4}\right) \quad \text { for } r_{1}<r<1 .
$$

Now by $[7 ; p .12]$ (and (5)), there exists $K_{2}>0$ such that

$$
\log ^{+}|1 / \Phi(z)| \leqslant K_{2}\left((1-r)^{-(\alpha+2)}\right) \log \left((1-r)^{-1}\right) \quad \text { on }|z|=r
$$

if $\frac{1}{2} \leqslant r<1$ and $r \notin F_{1}$. Since $Q$ is a polynomial all of whose roots lie in the unit disk, there exist $K_{3}>0$ and $r_{2}$ in $[0,1)$, such that

$$
|Q(z)| \geqslant K_{3} \quad \text { for } r_{2} \leqslant|z| \leqslant 1 \text {. }
$$

In view of (12), (13) and (14), it follows from (11) that there exists $r_{3}$ in $[0,1)$ such that the conclusion (4) holds (with $K=K_{1}+1$ ) on $|z|=r$ if $r \in\left[r_{3}, 1\right.$ ) and $r \notin F_{1}$. Taking $F$ to be the union of $F_{1}$ and $\left[0, r_{3}\right]$, it follows from (7) that conclusion (3) is also satisfied and so the proof of Lemma $A$ is complete. 


\section{5. - Proof of the Theorem (Section 2).}

Let $\alpha$ be the maximum of the orders of all the coefficients $f_{k j}(z)$, and as in the statement of the theorem, let $d>\alpha$ and set $\varepsilon=(d-\alpha) / 3$. Let $y_{0}(z)$ be an analytic solution in the disk of $\Omega\left(z, y, y^{\prime}\right)=0$. We may assume that $y_{0}(z)$ is of infinite order in the disk since (2) is immediate if it is of finite order.

Now set $p=\max \left\{k+j: f_{k j} \neq \equiv 0\right\}$ and $m=\max \left\{j: f_{p-i, j} \neq 0\right\}$, and let $I$ be the set of all $(k, j)$ such that $k+j<p$ and $f_{k j} \neq \equiv$. By dividing the relation $\Omega\left(z, y_{0}(z)\right.$, $\left.y_{0}^{\prime}(z)\right) \equiv 0$ through by $\left(y_{0}(z)\right)^{p}$, we can write this relation in the form,

$$
\Lambda(z) \equiv-\sum_{(k, j) \in I} f_{k j}(z)\left(y_{0}^{\prime}(z) / y_{0}(z)\right)^{j}\left(y_{0}(z)\right)^{k+j-p},
$$

where

$$
A(z)=\sum_{j=0}^{m} f_{p-j, j}(z)\left(y_{0}^{\prime}(z) / y_{0}(z)\right)^{i}
$$

Also, let $q=1+$ (cardinality of $I$ ).

Now since each $f_{k j}$ is of order $\leqslant \alpha$, there exists $r_{1}$ in $[0,1)$ such that for each $(k, j)$,

$$
\left|f_{k j}(z)\right| \leqslant \exp \left((1-r)^{-\alpha+1)}\right) \quad \text { on }|z|=r \text { if } r_{1} \leqslant r<1
$$

Furthermore, by Lemma $A$, there exist a positive constant $K$ and a subset $F$ of $[0,1)$ such that (3) holds and

$$
\left|f_{p-m, m}(z)\right| \geqslant \exp \left(-K /(1-r)^{\alpha+4}\right) \quad \text { on }|z|=r
$$

if $r$ belongs to $[0,1)$ and $r \notin F$.

We now prove,

Lemma B. - There exists $r_{2}$ in $\left[r_{1}, 1\right)$ such that if $r$ belongs to $\left[r_{2}, 1\right)$ and $r \notin F$, then for any point $z_{0}$ on $|z|=r$ at which

$$
\left|y_{0}^{\prime}\left(z_{0}\right) / y_{0}\left(z_{0}\right)\right| \geqslant \exp \left((1-r)^{-\sigma}\right) \quad \text { where } \sigma=\alpha+4+\varepsilon,
$$

we have

$$
\left|y_{0}\left(z_{0}\right)\right| \leqslant(m+1) q\left|y_{0}^{\prime}\left(z_{0}\right) / y_{0}\left(z_{0}\right)\right|^{p}
$$

Proof. - Since $\varepsilon>0$, clearly there exists $r_{2}$ in $\left[r_{1}, 1\right)$ such that for $r_{2} \leqslant r<1$, we have

$$
\exp \left((1-r)^{-(\alpha+1)}+K(1-r)^{-(\alpha+4)}\right)<(1 /(m+1)) \exp \left((1-r)^{-\sigma}\right) .
$$


We assert that this $r_{2}$ suffices for the lemma. To see this, let $r$ belong to $\left[r_{2}, 1\right)$ and $r \notin F$. Let $z_{0}$ be a point on $|z|=r$ for which (19) holds. If $\left|y_{0}\left(\tilde{z}_{0}\right)\right| \leqslant 1$, then in view of (19), clearly (20) holds. Hence we may assume,

$$
\left|y_{0}\left(z_{0}\right)\right|>1 \text {. }
$$

Furthermore, by (15), we have,

$$
\left|A\left(z_{0}\right)\right| \leqslant\left.\sum_{(k, j) \in I}\left|f_{k j}\left(z_{0}\right)\right|\left|y_{0}^{\prime}\left(z_{0}\right)\right| y_{0}\left(z_{0}\right)\right|^{j}\left|y_{0}\left(z_{0}\right)\right|^{k+j-p} .
$$

We now distinguish two cases.

Case I: $m=0$. Then $A\left(z_{0}\right)=f_{p-n, m}\left(z_{0}\right)$, so by (18),

$$
\Lambda\left(z_{0}\right) \geqslant \exp \left(-K /(1-r)^{2+4}\right)
$$

In view of (23) and (24), I must be non-empty, and it is clearly impossible that each term in the sum on the right side of $(23)$ be $<(1 / q)\left|\Lambda\left(z_{0}\right)\right|$. Hence for some $(k, j)$ in $I$ (depending on $z_{0}$, of course), we must have,

$$
\left.\left|f_{k j}\left(z_{0}\right)\right|\left|y_{0}^{\prime}\left(z_{0}\right)\right| y_{0}\left(z_{0}\right)\right|^{i}\left|y_{0}\left(z_{0}\right)\right|^{k+j-p} \geqslant(1 / q)\left|\Lambda\left(z_{0}\right)\right|
$$

Since $k+j \leqslant p-1$, it follows from (22) that $\left.\left|y_{0}\left(z_{0}\right)^{-1} \geq\right| y_{0}\left(z_{0}\right)\right|^{k+j-p}$. Hence in view of the estimates (17) and (24), and the condition (21) on $r$, it follows from (25) that,

$$
\left.\exp \left((1-r)^{-\sigma}\right)\left|y_{0}^{\prime}\left(z_{0}\right)\right| y_{0}\left(z_{0}\right)\right|^{j}\left|y_{0}\left(z_{0}\right)\right|^{-1} \geqslant(1 / q) .
$$

But then by assumption (19) and the fact that $j+1 \leqslant p$, we clearly obtain (20) for $m=0$ so this case is proved.

Case II: $m>0$. We may write,

$$
\mathcal{A}\left(z_{0}\right)=f_{p-m, n}\left(z_{0}\right)\left(\left(y_{0}^{t}\left(z_{0}\right) / y_{0}\left(z_{0}\right)\right)^{m}\left(1+\sum_{j=0}^{m-1} \Psi_{j}\left(z_{0}\right)\right),\right.
$$

where

$$
\Psi_{j}\left(z_{0}\right)=\left(f_{p-j, j}\left(z_{0}\right) / t_{p-m, m}\left(z_{0}\right)\right)\left(y_{0}^{\prime}\left(z_{0}\right) / y_{0}\left(z_{0}\right)\right)^{j-m} \quad \text { for } j<m \text {. }
$$

Since $j-m \leqslant-1$, it easily follows from the assumption (19), the estimates (17) and (18), and the condition (21) on $r$, that $\left|\Psi_{j}\left(z_{0}\right)\right|<(1 /(m+1))$ for $j<m$. Hence from $(27),\left|A\left(z_{0}\right)\right| \geqslant\left((1 /(m+1))\left|f_{p-m, m}\left(z_{0}\right)\right|\left|y_{0}^{\prime}\left(z_{0}\right) / y_{0}\left(z_{0}\right)\right|^{m}\right.$, which in view of (18) and (19) (and the facthat $m \geqslant 1$ in this case) yields,

$$
\left|A\left(z_{0}\right)\right| \geqslant(1 /(m+1)) \exp \left(-K(1-r)^{-(\alpha+4)}+(1-r)^{-\sigma}\right) \text {. }
$$


As in Case I, we see from (23) and (29) that $I$ must be non-empty and that it is impossible for each term on the right side of (23) to be $<(1 / q)\left|\Lambda\left(z_{0}\right)\right|$. Hence for some $(k, j)$ in $I$ (depending on $z_{0}$ ), we must again have the relation $(25)$. From this it follows (using the estimates (17) and (29), the condition (21) on $r$ and (22)) that

$$
\left.(1 /(m+1)) \mid y_{0}^{\prime}\left(z_{0}\right) / y_{0}\left(z_{0}\right)\right\}^{\prime}\left|y_{0}\left(z_{0}\right)\right|^{-1} \geqslant(1 /(m+1) q)
$$

From assumption (19) and the fact that $j<p$. we then obtain (20) which proves Lemma $B$ in Case II also.

Continuing with the proof of the main result, we now define for $r \in\left[r_{2}, 1\right.$ ) and $r \notin F$, the set

$$
D_{r}=\left\{z:|z|=r \text { and }\left|y_{0}^{\prime}(z)\right| y_{0}(z) \mid<\exp \left((1-r)^{-\sigma}\right)\right\}
$$

Let $C_{r}$ denote the complement of $D_{r}$ with respect to the circle $|z|=r$. Now if $z$ belongs to $C_{r}$, then by Lemma $\mathrm{B},(20)$ holds for $z$ and hence,

$$
\log ^{+}\left|y_{0}(z)\right| \leqslant K_{1}+p \log ^{\dagger}\left|y_{0}^{\prime}(z)\right| y_{0}(z) \mid
$$

where $K_{1}=\log ^{+}(m+1) q$. Writing $z=r \exp (i \theta)$ and letting $C_{r}^{*}=\{\theta: \theta \leqslant \theta \leqslant 2 \pi$ and $\left.r \exp (i \theta) \in C_{r}\right\}$, we have from (32),

$$
\int_{\partial_{r}^{*}} \log ^{+}\left|y_{0}(r \exp (i \theta))\right| d \theta \leqslant K_{1} 2 \pi+p \int_{\partial_{\hat{r}}} \log ^{+}\left|y_{0}^{\prime}(r \exp (i \theta))\right| y_{0}(r \exp (i \theta)) \mid d \theta .
$$

The second term on the right of $(33)$ is at most $(2 \pi p) m\left(r, y_{0}^{\prime} / y_{0}\right)$. Now by $[5$; p. 246], there exist a positive constant $K_{2}$ and a subset $F_{1}$ of $[0,1$ ) such that $\int_{F_{1}}(1-r)^{-2} d r\left(\right.$ and hence $\left.\int_{F_{1}}(1-r)^{-1} d r\right)$ is finite, and such that for $r \notin F_{1}$,

$$
m\left(r, y_{0}^{\prime} / y_{0}\right) \leqslant K_{2}\left(\log \left((1-r)^{-1}\right)+\log T\left(r, y_{0}\right)\right) .
$$

Now by assumption, $y_{0}$ is of infinite order and hence $T\left(r, y_{0}\right) \rightarrow+\infty$ as $r \rightarrow 1$ (see $\left[4 ;\right.$ p. 140]). Thus clearly, there exists $r_{3}$ in $\left[r_{2}, 1\right)$ such that for $r_{3} \leqslant r<1$, we have $p K_{2} \log T\left(r, y_{0}\right) \leqslant\left(\frac{1}{2}\right) T\left(r^{*}, y_{0}\right)$ and $K_{1} \leqslant p K_{2} \log \left((1-r)^{-1}\right)$. Dividing (33) by $2 \pi$, and using $(34)$ and the above inequalities, we clearly obtain,

$$
(1 / 2 \pi) \int_{\sigma_{r}^{*}} \log ^{+}\left|y_{0}(r \exp (i \theta))\right| d \theta \leqslant 2 p K_{2} \log \left((1-r)^{-1}\right)+T\left(r, y_{0}\right) / 2,
$$

for $r_{3} \leqslant r<1$ and $r \notin E$, where $E$ is the union of $F$ and $F_{2}$. Since (3) holds for $F$ and $F_{1}$, we have

$$
\int_{E}(1-r)^{-1} d r<+\infty
$$


Now let $D_{r}^{*}=\left\{\theta: 0 \leqslant \theta \leqslant 2 \pi\right.$ and $\left.r \exp (i \theta) \in D_{r}\right\}$. Adding

$$
(1 / 2 \pi) \int_{D_{r}^{*}} \log ^{+}\left|y_{0}(r \exp (i \theta))\right| d \theta
$$

to both sides of (35) and noting that the left side then becomes $m\left(r, y_{0}\right)$ which is $T\left(r, y_{0}\right)$ since $y_{0}$ is analytic, we obtain,

$$
T\left(r, y_{0}\right) \leqslant 4 p K_{2} \log \left((1-r)^{-1}\right)+(1 / \pi) \int_{D_{r}^{*}} \log ^{+}\left|y_{0}(r \exp (i \theta))\right| d \theta,
$$

for $r_{3} \leqslant r<1$ and $r \notin E$.

In the following Lemma, we estimate the integrand appearing on the right side of $(37)$.

Lemma C. - There exists $r_{4}$ in $\left[r_{3}, 1\right)$, such that

$$
\left|y_{0}(z)\right| \leqslant \exp \left(\exp \left[(1-r)^{-(\sigma+\theta)}\right]\right) \quad \text { for all } z \text { in } D_{r},
$$

if $r_{4} \leqslant r<1$ and $r \notin E$.

Proof. - Let $\delta$ be a number in $(0,1)$ such that $y_{0}(\approx)$ has no zeros for $0<|z| \leqslant \delta$. By JeNSEN's formula [5; p. 166[, there is a constant $\lambda>0$ such that for $0 \leqslant r<1$,

$$
T\left(r, 1 / y_{0}\right)=T\left(r, y_{0}\right)+h(r), \quad \text { where }|h(r)| \leqslant \lambda
$$

Since $\varepsilon>0$, there exists $r_{4} \geqslant \delta$ in $\left[r_{3}, 1\right)$ such that for $r_{4} \leqslant r<1, \exp \left((1-r)^{-(\sigma+\varepsilon)}\right)$ is greater than both of the following:

$$
\log (m+1) q+p(1-r)^{-\sigma}+4 \pi \exp \left((1-r)^{-\sigma}\right),
$$

and

$$
(2(1-\delta) / \delta) \exp \left((1-r)^{-\sigma}\right)+2 \lambda+2\left|N\left(\delta, 1 / y_{0}\right)\right|
$$

We assert that $r_{4}$ satisfies the conditions of the lemma. To prove this, let $r$ belong to $\left[r_{4}, 1\right)$ and let $r \notin E$. We distinguish two cases.

Case $I: C_{r}$ is not empty.

Let $z_{0}$ be an element of $D_{r}$, say $z_{0}=r \exp \left(i \theta_{0}\right)$. Let $\theta_{1}$ be the infimum of the set of all $\theta$ in $\left[\theta_{0}, \theta_{0}+2 \pi\right)$ for which $r \exp (i \theta)$ belongs to $C_{r}$, and let $z_{1}=r \exp \left(i \theta_{1}\right)$. It is easily verified (using (31)) that

$$
\left.\left|y_{0}^{\prime}\left(z_{1}\right) / y_{0}\left(z_{1}\right)\right|=\exp \left((1-r)^{-\sigma}\right) \quad \text { (so } \theta_{0}<\theta_{1}<\theta_{0}+2 \pi\right)
$$


and that for $\theta_{0} \leqslant \theta<\theta_{1}, r \exp (i \theta)$ belongs to $D_{r}$. Hence if we let $\Gamma$ denote the arc $z=r \exp (-i \varphi),-\theta_{1} \leqslant \varphi \leqslant-\theta_{0}$, then

$$
\mid y_{0}^{\prime}(z) / y_{0}(z) \leqslant \exp \left((1-r)^{-\sigma}\right) \quad \text { on } \Gamma \text {. }
$$

Thus clearly, $y_{0}$ is nowhere zero on the are $\Gamma$, and it easily follows that $y_{0}$ is nowhere zero on some simply-connected neighborhood of $\Gamma$. Hence there exists an analytic branch $g$ of $\log y_{0}$ on this neighborhood. Since $g^{\prime}=y_{0}^{\prime} / y_{0}$, we have

$$
g\left(z_{0}\right)-g\left(z_{1}\right)=\int_{\eta^{\prime}}\left(y_{0}^{\prime}(\zeta) / y_{0}(\zeta)\right) d \zeta
$$

Taking the exponential of (44), and using (43), we obtain,

$$
\left|y_{0}\left(z_{0}\right)\right| \leqslant\left|y_{0}\left(z_{1}\right)\right| \exp \left(2 \pi \exp \left((1-r)^{-\sigma}\right)\right)
$$

But by (42) and Lemma $B$ (applied to $z_{1}$ ), we have

$$
\left|y_{0}\left(z_{1}\right)\right| \leqslant(m+1) q \exp \left(p(1-r)^{-\sigma}\right)
$$

This inequality, together with (45) and (40), yields $\left|y_{0}\left(z_{0}\right)\right| \leqslant \exp \left(\exp (1-r)^{-(\sigma+\delta)}\right)$ which is (38) for $z=z_{0}$. Since $z_{0}$ was an arbitrary element of $D_{r}$, we have obtained the desired conclusion in Case I.

Case $I I: C_{r}$ is empty.

In this case, assume that (38) fails to hold, i.e., there exist a point $z_{0}=r \exp \left(i \theta_{0}\right)$ in $D_{r}$ such that,

$$
\left|y_{0}\left(z_{0}\right)\right|>\exp \left(\exp \left[(1-r)^{-(\sigma+8)}\right]\right)
$$

Now let $z_{1}=r \exp \left(i \theta_{1}\right)$ (where $\left.\theta_{0}<\theta_{1}<\theta_{0}+2 \pi\right)$ be any point on $|z|=r$ distinct from $z_{0}$, and let $\Gamma$ be the arc, $\zeta=r \exp (-i \varphi),-\theta_{1} \leqslant \varphi \leqslant-\theta_{0}$. Since all points $|z|=r$ belong to $D_{r}$ in this case, (43) holds on $\Gamma$. Hence $y_{0}$ is nowhere zero on $\Gamma$ and as in case $I$, there is a branch $g$ of $\log y_{0}$ in a neighborhood of $I$. Thus (44) and hence (45) hold. By (47) we therefore obtain,

$$
\left.\left|y_{0}\left(z_{1}\right)\right| \geqslant \exp \left(\exp [1-r)^{-(\sigma+\varepsilon)}\right]-2 \pi \exp \left[(1-r)^{-\sigma}\right]\right)
$$

But by $(40), \exp \left((1-r)^{-(\sigma+\varepsilon)}\right) \geqslant 4 \pi \exp \left((1-r)^{-\pi}\right)$, and hence,

$$
\left|y_{0}\left(z_{1}\right)\right| \geqslant \exp \left(\frac{1}{2} \exp (1-r)^{-(\sigma+\varepsilon)}\right)
$$


By (47), this inequality holds for $z_{1}=z_{0}$ also, so (49) is valid for all points $z_{1}$ on $|z|=r$. Hence clearly,

$$
m\left(r, y_{0}\right) \geqslant \frac{1}{2} \exp \left((1-r)^{-(\alpha+\bar{\varepsilon})}\right)
$$

and

$$
m\left(r, 1 / y_{0}\right)=0
$$

Now let $n(t)$ denote the number of poles (counting multiplicity) of $1 / y_{0}$ in $|z| \leqslant t$. Since $y_{0}$ is analytic, we have by the argument principle,

$$
n(r)=(1 / 2 \pi i) \int_{|z|=r}\left(y_{0}^{\prime}(z) / y_{0}(z)\right) d z
$$

Since all points on $|z|=r$ are elements of $D_{r}$ in this case, it follows that,

$$
n(r) \leqslant \exp \left((1-r)^{-\sigma}\right)
$$

By assumption concerning $\delta$ (and since $r<1$ ), we have,

$$
\left|N\left(r, 1 / y_{0}\right)\right| \leqslant \int_{\delta}^{r}[(n(t)-n(0)) / t] d t+n(0) \log (1 / r)
$$

and hence,

$$
\left|N\left(r, 1 / y_{0}\right)\right| \leqslant((1-\delta) / \delta) \exp \left((1-r)^{-\sigma}\right)+\left|N\left(\delta, 1 / y_{0}\right)\right|
$$

But by (39) (and the fact that $y_{0}$ is analytic), we have $m\left(r, y_{0}\right) \leqslant\left\{T\left(r, 1 / y_{0}\right)\right\}+\lambda$. However, in view of $(50),(51),(54)$ and the condition $(41)$ on $r$, this is easily seen to be impossible. This contradiction proves the desired conclusion in Case II, and hence the lemma is proved.

We now conclude the proof of the main theorem. In view of (37) and Lemma $\mathrm{C}$, it easily follows that there exists $r_{5}$ in $\left(r_{4}, 1\right)$ such that

$$
T\left(r, y_{0}\right) \leqslant 3 \exp \left((1-r)^{-(\sigma+\delta)}\right)
$$

if $r_{5} \leqslant r<1$ and $r \notin E$. Let $A$ be the union of $E$ and $\left[0, r_{5}\right]$. In view of (36), the integral $A=\int_{A}(1-r)^{-1} d r$ is finite. We now proceed to eliminate the possible exceptional set in $(55)$, by proving that if $\gamma=A+1$, then for all $r$ in $[0,1)$,

$$
T\left(r, y_{0}\right) \leqslant 3 \exp \left(c^{\gamma(\sigma+\varepsilon)}(1-r)^{-(\sigma+\varepsilon)}\right)
$$


To prove this, let $r$ belong to $[0,1)$, and set $\beta=\beta(r)=(1-r)\left(1-e^{-\gamma}\right)$. Then $[r, r+\beta]$ lies in $[0,1)$ and it is easily verified that the integral of $(1-r)^{-1}$ over $[r, r+\beta]$ is $\gamma$. Since $\gamma>\Delta$, clearly $[r, r+\beta]$ cannot be contained in $A$. Hence there exists $t$ in $[r, r+\beta]$ with $t \notin A$. By (55),

$$
T\left(t, y_{0}\right) \leqslant 3 \exp \left((1-t)^{-(\sigma+\varepsilon)}\right) .
$$

Since $T$ is increasing and since $1-t \geqslant e^{-\gamma}(1-\gamma),(56)$ now follows immediately from $(57)$.

From (56) and $\left[4 ;\right.$ p. 24], it now follows easily that there exists $r_{0}$ in $[0,1)$ such that

$$
\log ^{+} M\left(r, y_{0}\right) \leqslant \exp \left((1-r)^{-(\sigma+n \varepsilon)}\right)
$$

for all $r$ with $r_{0} \leqslant r<1$. Since $\sigma+2 \varepsilon=d+4$, we have thus obtained the conclusion (2) of the main theorem and so the proof is complete.

6. - For meromorphic solutions, we have the following result:

Corollary. - Let $h(\approx)$ be a meromorphic function in the unit disk which satisfies a first order algebraic differential equation whose coefficients are analytic functions of finite order in the disk. Then for some positive constant $b$, the inequality $T(r, h) \leqslant$ $\leqslant \exp \left((1-r)^{-b}\right)$ holds on some interval $\left[r_{0}, 1\right)$ (where $r_{0}<1$ ), if either of the following two conditions is satisfied:

(4) For some meromorphic function $\varphi(z)$ of finite order in the disk, the sequence of zeros of $h(z)-\varphi(z)$ has a finite convergence exponent.

$B$ ) The sequence of poles of $h(z)$ has a finite convergence exponent.

Proof. - In Case $(A)$, we can write $\left[7 ;\right.$ p. 11], $\varphi=\varphi_{1} / \varphi_{2}$ where $\varphi_{1}$ and $\varphi_{2}$ are analytic and of finite order in the disk. Let $\psi$ be the canonical product $[7 ; p .7]$ whose sequence of zeros is the sequence of zeros of $h-\varphi$. Then $\psi$ is analytic and of finite order in the disk $[7 ;$ p. 8], and clearly the function

$$
w(z)=\psi(z) /\left(h(z)-\left[\varphi_{1}(z) / \varphi_{2}(z)\right]\right)
$$

is analytic in the disk. Since $h$ satisfies a first order equation whose coefficients are of finite order, it easily follows that the same is true of the analytic function $w(z)$. Hence by the main result (§2), for some $a>0$, the inequality $T(r, w) \leqslant$ $=\exp \left((1-r)^{-a}\right)$ holds on some interval $\left[r_{1}, 1\right)$ (where $r_{1}<1$ ). In view of $(59)$ and the fact that $\psi, \varphi_{1}$ and $\varphi_{2}$ are of finite order, the conclusion of the corollary now follows immediately using the usual calculational rules for the characteristic $[4 ; p .15]$. 
In Case $(B)$, we form the canonical product $\psi(z)$ whose zeros are the poles of $h$. Then $w=\psi h$ is analytic and the proof proceeds as in Case $(A)$.

REMARK. - As mentioned in $\S 1$, there is no uniform growth estimate for arbitrary meromorphic solutions of equations in the class treated in this paper, since any preassigned function $\Phi(r)$ can be dominated at a sequence of $r$ tending to 1 , by the NEVANLINNA characteristic of such a solution in the disk. The details of the construction of such solutions are very similar to those in [3], where the construction was carried out for the plane, and hence we omit them. However, for the reader's convenience, we will briefly sketch the construction. We take a function $h(z)$ which is the quotient of two Weierstrass-type infinite products $\varphi_{1}$ and $\varphi_{2}$, each analytic in the disk, where the zeros of $\varphi_{1}$ and $\varphi_{2}$ are as follows: Let $\left(r_{1}, r_{2}, \ldots\right)$ be a sequence of distinct real numbers in $(1 / 2,1)$, which converges to 1 and has a finite convergence exponent. For each member $r_{n}$ of this sequence, let $\varphi_{1}$ and $\varphi_{2}$ have zeros $z_{n}$ and $w_{n}$ respectively on $|z|=r_{n}$, both of the same high multiplicity $\mu_{n}$, and such that $z_{n}$ and $w_{n}$ are very close together but distinct $\left(i . e ., 0<\left|z_{n}-w_{n}\right|<\varepsilon_{n}\right)$. If the sequence $\left\{\mu_{n}\right\}$ has a sufficiently rapid increase (i.e., $\left.\mu_{n}>\left[2 /\left(1-r_{n}\right)\right] \Phi\left[\left(1+r_{n}\right) / 2\right]\right)$, then the characteristic of the quotient $h=\varphi_{1} / \varphi_{2}$ will dominate $\Phi(r)$ at the sequence $\left\{\left(1+r_{n}\right) / 2\right\}$. On the other hand, by taking the sequence $\left\{\varepsilon_{n}\right\}$ to have a sufficiently rapid decrease, we can insure that the logarithmic derivative $h^{\prime} / h$ will be of finite order in the disk. (To see this, we first note that $N\left(r, h^{\prime} / h\right)$ offers no problem since the convergence exponent of $\left\{r_{n}\right\}$ is finite (see $\left[4 ;\right.$ p. 139]). Since $h^{\prime} / h=\left\langle\varphi_{1}^{\prime} / \varphi_{1}\right)-\left(\varphi_{2}^{\prime} / \varphi_{2}\right)$, it can be shown that by taking $z_{n}$ sufficiently close to $w_{n}, m\left(r, h^{\prime} / h\right)$ will offer no problem except possibly for small $r$-intervals $I_{n}$ around the $r_{n}$ (very similar to those appearing in the proof of Lemma $A$ ), whose union $F$ satisfies $\int_{F^{\prime}}(1-r)^{-1} d r<+\infty$. The exceptional set $F$ for $T\left(r, h^{\prime}(h)\right.$ is then handled as in the proof of our main result here.) Since $h^{r} / h$ is of finite order in the disk, $h$ is a solution of an equation $f_{1} h^{\prime}-f_{2} h=0$, where $f_{1}$ and $f_{2}$ are analytic functions of finite order in the disk [7; p. 11].

\section{BIBLIOGRAPHY}

[1] S. BANK, On the growth of solutions of algebraic differential equations whose coefficients ait arbitrary entire functions, Nagoya Math. J., 39 (1970), pp. 107-117.

[2] S. BANK, A result concerning meromorphic solutions in the unit dist of algebraic differential equations, Comp. Math, 22 (1970), pp. 367-381.

[3] S. BANK, A note on algebraio differential equations whose coefficients are entire functions of finite order, to appear.

[4] R. Nevancinna, Le Théorème de Picard-Bonel et la théorie des fonctions méromorphes, Gauthier-Villars, Paris, 1929. 
[5] R. Nevandinna, Analytie functions, Springer-Verlag, New York, 1970 (English trans.).

[6] S. SaKs - A. ZYGMund, Analytic funetions, Monografie Mat. (Englsh transl.), Tom 28, Warsaw, 1952.

[7] M. TsuJ, Canonical product for a meromorphic function in a unit circles, J. Math. Soc. Japan, 8 (1956), pp. 7-21.

[8] G. Valmon, Fonctions analytiques et équations différentielles, J. Math. pures et appl., 31 (1952), pp. 292-303.

[9] G. VaLIRon, Leetures on the general theory of integral functions, Edouard Privat, Toulouse, 1923. 